\title{
Relation entre la littératie familiale en milieu francophone et l'incidence d'un programme de maternelle quatre ans à temps plein sur le développement du langage et de la lecture des enfants
}

\author{
Claire Maltais \\ Université d'Ottawa
}

\begin{abstract}
Résumé
Cette recherche s'inscrit dans le cadre des littératies multiples. Elle s'intéresse à deux de ses composantes, les littératies scolaire et familiale en milieu minoritaire. Elle porte sur les effets d'un programme de maternelle quatre ans à temps plein sur le langage et la lecture d'élèves dont le milieu familial démontre un haut niveau d'utilisation du français (HNUF) dans ses activités de littératie, comparativement à des élèves dont le milieu familial utilise peu le français lors de ses activités (FNUF). Les résultats indiquent qu'au niveau du langage, le programme produit peu d'effets chez les élèves HNUF alors que chez les élèves FNUF, il produit une amélioration significative à la fin de la maternelle et de la $2^{\mathrm{e}}$ année, sans toutefois permettre aux élèves FNUF de rejoindre les élèves HNUF. En lecture, les gains significatifs observés chez les deux groupes à la fin de la maternelle se maintiennent en $2^{\mathrm{e}}$ année. Le programme de maternelle à temps plein compense donc, en partie, le manque d'activités de littératie en français de la famille.
\end{abstract}

\section{Abstract}

This research was conducted within the multiple literacies theoretical framework. It focuses on two of its components, school and family literacies in a French minority setting. It investigates the effects of a full time kindergarten program for four year old children on language and reading skills. It compares pupils from families using a high level of French in their home literacy activities (HNUF) to pupils from families using a low level of French in their home literacy activities (FNUF). Results on French language skills show that the program has few effects on pupils from the HNUF group, but produces significant gains on pupils from the FNUF group, as measured at the end of kindergarten and of second grade. However, language skills of pupils from the FNUF group do not reach the level obtained by those from the HNUF group. As for reading skills, the significant gains observed on both groups at the end of kindergarten are maintained at the end of second grade. Therefore, the full time kindergarten program is compensating in part for the low level of French in home literacy activities. 


\section{Introduction}

Cette recherche s'inscrit dans le cadre des littératies multiples. Selon Masny (2001), les littératies se composent de mots, de gestes, d'attitudes, de manières de parler, d'écrire et de valoriser les réalités de la vie, et renvoient à un construit social. Elles comprennent les littératies personnelle, communautaire, scolaire et critique. La présente recherche s'intéresse plus particulièrement à la littératie scolaire et à la littératie familiale comprise dans la littératie communautaire.

En Ontario, le français est une langue minoritaire. La population étudiante des écoles de langue française est très variée sur le plan langagier. Elle est composée d'enfants qui parlent le français mais dont le niveau de langue varie beaucoup, d'enfants de parents « ayants droit » dont certains parlent peu ou pas du tout français, mais qui ont le droit de fréquenter les écoles de langue française, et d'autres enfants pour qui le français est une troisième langue (clientèle multiethnique). Pour plusieurs de ces enfants, les activités de littératie en milieu familial se font dans une langue autre que le français. Ils arrivent donc à l'école avec des façons de parler, d'écouter et d'agir qui sont différentes selon leurs cultures. Pour certains, la littératie personnelle se rapproche de celle qui est véhiculée par le système scolaire alors que pour d'autres, il existe des différences majeures entre, d'une part, ce qui est requis au préscolaire ou à l'école et, d'autre part, ce qui est exigé à maison et dans la communauté (Comber et Cormack, 1997).

Le préscolaire étant une étape de transition entre la famille et l'école, l'un des conseils scolaires de langue française en Ontario a mis sur pied un programme de maternelle quatre ans à temps plein en septembre 2000. ॥ espérait ainsi favoriser la réussite scolaire de ses élèves en leur permettant de fréquenter, dès l'âge de quatre ans, un milieu de vie qui préconise le développement de la langue et de la culture françaises. Cette étude vise donc à répondre à la question suivante : est-ce qu'un programme de maternelle quatre ans à temps plein peut compenser une faible utilisation du français dans les activités de littératie familiale? Pour répondre à cette question, la présente recherche compare le développement du langage et de la lecture d'un groupe d'élèves dont le milieu familial démontre un haut niveau d'utilisation du français dans ses activités de littératie à celui d'un groupe d'élèves dont le milieu familial utilise peu le français lors de ses activités.

\section{Contexte de la recherche}

La recension des écrits examine les recherches qui ont évalué les effets des programmes préscolaires et de la littératie familiale sur le développement du langage et de la lecture des enfants. 


\section{Effets des programmes préscolaires sur le langage et la lecture des enfants}

La plupart des recherches menées sur les effets des programmes préscolaires sur le rendement des enfants ont été faites dans des services de garde, puisque les programmes de maternelle à temps plein pour les enfants de quatre ans sont peu répandus dans les systèmes scolaires au Canada et aux États-Unis. Les résultats de plusieurs d'entre elles doivent être considérés avec prudence en raison de la qualité variable des services de garde évalués (accrédités et non-accrédités) et de la qualification des éducateurs œuvrant auprès des enfants dans ces services de garde. De plus, certaines recherches n'évaluent qu'un domaine de développement (p. ex., l'aspect cognitif) et peu d'entre elles comportent une évaluation du programme à moyen ou à long terme.

Néanmoins, sur le plan langagier, bon nombre de ces études confirment qu'il y a une relation positive à court terme entre les programmes préscolaires de qualité et le développement du langage (Maltais, 2005; Maltais, Herry et Levesque, 2001; Palacio-Quentin et Coderre, 1999; Tremblay, 2003). D'autres concluent que si cette relation n'apparaît pas à court terme, elle peut se manifester ultérieurement (Maltais et Herry, 2005; Wessels, Lamb et Hwang, 1996).

Pour le rendement en lecture, les résultats de plusieurs recherches semblent indiquer que les programmes préscolaires ont des effets sur cette composante, mais que l'ampleur et la nature de ces effets sont modulées par des variables comme le niveau socioéconomique et l'appartenance à une minorité visible ou raciale (Jarousse, Mingat et Richard, 1992; Jeantheau et Murat, 1998; Wessels, Lamb et Hwang, 1996). Les enfants des milieux défavorisés et ceux appartenant à une minorité visible ou raciale semblent bénéficier davantage des programmes préscolaires que les enfants de classe moyenne ou aisée (O'Brien Caughy, DiPietro et Scrobino, 1994).

Les résultats de ces recherches permettent d'avancer l'hypothèse que les programmes préscolaires de qualité ont des effets positifs sur le développement langagier et sur le rendement en lecture des enfants provenant des milieux défavorisés et de ceux appartenant à une minorité visible ou raciale, et que si ces effets n'apparaissent pas à court terme, ils sont présents à plus long terme. On peut alors se demander si ces résultats peuvent s'appliquer à une population d'enfants dont les activités de littératie familiale se passent dans une langue autre que celle de l'enseignement.

\section{Effets de la littératie familiale sur le langage et la lecture des enfants}

L'influence du milieu familial sur le développement du langage et de la lecture chez les jeunes enfants apparaît déterminante. Certains auteurs affirment que les habiletés langagières des enfants d'âge préscolaire prédisent la réussite en lecture au début du primaire (Walker, Greenwood, Hart et Carta, 1994). D'autres précisent que les élèves qui ont acquis, avant leur entrée en première année, des habiletés langagières (vocabulaire, conscience phonologique, etc.) et des habiletés en conscience de l'écrit (aspects fonctionnels et formels de l'écrit) sont plus susceptibles d'obtenir un bon rendement en lecture (Adams, 1990). Ces habiletés développées au cours de l'enfance seraient prédictives du succès scolaire ultérieur (Burns, Espinosa et Snow, 2003; Walker, Greenwood, Hart et Carta, 1994). 
Parmi les activités de littératie familiale, la lecture de livres aux enfants fait l'objet de plusieurs études qui démontrent des effets sur le développement du vocabulaire, sur la connaissance des aspects fonctionnels (les buts de la lecture et de l'écriture) et des aspects formels de l'écrit (l'acquisition de la connaissance de la lettre et du mot, l'orientation de la lecture, etc.), ainsi que sur la syntaxe et la structure du récit (Bus van ljzendoorn et Pellegrini, 1995; Giasson, 2003; Scarborough et Dobrich, 1994b; Thériault, 1995; Weigel, Martin et Bennett, 2005). De plus, la fréquence des lectures faites aux enfants est aussi associée à leur rendement futur en lecture (Bus, Van ljzendoorn et Pellegrini, 1995).

Par contre, d'autres chercheurs affirment que la relation entre la lecture faite à haute voix par le parent et le rendement des enfants en lecture est plus faible que ne le suppose la plupart des recherches (Sénéchal, Thomas et Monker, 1995; Whitehurst, Epstein, Angell, Payne, Crone et Fishel, 1994). Ce sont plutôt les interactions vécues entre l'adulte et l'enfant au cours de cette lecture qui sont positivement reliées au rendement en lecture lors des premières années scolaires (Enz, 2003; Meyer, Wardrop, Stahl et Linn, 1994; Sénéchal, Thomas et Monker, 1998).

Burgess, Hecht et Lonigan (2002) indiquent que plusieurs aspects de la littératie familiale peuvent influencer le développement de la littératie de l'enfant. Ces aspects sont : les caractéristiques démographiques et sociales de l'environnement familial, les activités parentales qui exposent l'enfant à des modèles d'utilisation de la littératie et les efforts des parents pour engager l'enfant dans des activités destinées à stimuler la littératie ou le langage oral. II semble que la littératie familiale, lorsqu'elle inclut des activités qui se rapprochent de celles vécues en milieu scolaire, a un effet positif sur l'apprentissage du langage et de la lecture chez les enfants.

\section{Objectifs de la recherche}

La majorité des recherches discutées précédemment ont été effectuées auprès d'enfants dont la langue maternelle est celle de la majorité, la langue parlée à la maison étant la même que celle utilisée à l'école. En contexte de langue française en milieu minoritaire, le niveau d'utilisation du français dans les activités de littératie familiale varie beaucoup, allant d'une utilisation constante du français à une utilisation constante d'une autre langue. Dans un tel contexte, le fait, pour les enfants de quatre ans de fréquenter un programme de maternelle à temps plein à l'école peut-il compenser une faible utilisation du français en milieu familial?

La présente étude a pour but, dans un premier temps, de vérifier les effets d'un programme de maternelle quatre ans à temps plein mis sur pied par un conseil scolaire de langue française en Ontario en septembre 2000 sur le développement du langage et de la lecture de deux groupes d'élèves : I'un dont le milieu familial démontre un haut niveau d'utilisation du français dans ses activités de littératie (HNUF) et l'autre dont le milieu familial utilise peu le français (FNUF). Dans un deuxième temps, si le programme produit des effets positifs sur le langage et la lecture, l'étude tentera de vérifier s'il permet aux élèves du groupe FNUF de rejoindre la performance des élèves du groupe HNUF.

\section{La méthodologie}

La méthodologie inclut la description du modèle de recherche, des sujets, de la procédure et des instruments de collecte des données, de même que des méthodes d'analyse de celles-ci. 


\section{Le modèle de recherche}

Pour vérifier les effets du programme, le modèle de recherche quasi-expérimental utilisé compare, au cours de la première étape, le développement global (langagier, scolaire, socio-affectif et psychomoteur) des élèves qui ont bénéficié du programme de maternelle quatre ans à temps plein au cours de l'année 2000-2001 à ceux qui ont fréquenté un programme semblable, mais à mi-temps, au cours de l'année 1999-2000. Au cours de la deuxième étape, les deux groupes sont à nouveau comparés à la fin de leur $2 \mathrm{e}$ année. Le choix de ce modèle s'est imposé parce qu'il n'était pas possible de former au hasard un groupe expérimental et un groupe témoin. En 2000-2001, tous les élèves du conseil scolaire visé par cette étude bénéficiaient du programme de maternelle à temps plein. De plus, la comparaison avec un groupe témoin provenant d'un autre conseil scolaire ontarien n'a pas été possible parce que leur population n'était pas comparable à celle du groupe cible, ou encore parce que les conseils scolaires avaient, eux aussi, un programme de maternelle à temps plein pour les enfants de quatre ans. Pour les besoins de cette étude, seuls les résultats en langage et en lecture sont utilisés.

Le modèle utilisé pour répondre à la deuxième question de recherche compare, à la fin de la maternelle et de la $2^{\mathrm{e}}$ année, la performance en langage et en lecture des élèves du groupe HNUF à la performance des élèves du groupe FNUF..

\section{Les sujets}

Les sujets se répartissent en deux groupes. Le premier comprend les élèves HNUF. Ces élèves proviennent de trois écoles de l'est d'Ottawa. Tel que présenté au tableau 1, à la fin de la maternelle, le groupe HNUF se subdivise en deux sous-groupes : le sous-groupe à mi-temps compte 92 élèves et le sous-groupe à temps plein, 116. À la fin de la $2^{e}$ année, ces deux sous-groupes comptent respectivement 61 et 98 élèves.

Le deuxième groupe comprend les élèves FNUF. Ils proviennent d'une école de l'ouest d'Ottawa. À la fin de la maternelle, le sous-groupe à mi-temps compte 40 élèves et le sous-groupe à temps plein, 30 . À la fin de la $2^{\mathrm{e}}$ année, les nombres de sujets dans ces sous-groupes se situent à 32 et 29 respectivement. Le nombre de sujets a diminué en raison de plusieurs facteurs, tels que les déménagements dans une autre ville ou une autre province, ou encore les départs vers une école d'un autre conseil scolaire.

Des analyses statistiques (analyse de variance Anova pour l'âge des sujets et des analyses Chi 2 pour les autres variables) n'ont pas trouvé de différence significative entre les sous-groupes au niveau du sexe des élèves, de leur appartenance à une minorité ethnique et du niveau d'éducation des parents.

Afin de s'assurer que les élèves inclus dans les groupes HNUF et FNUF ont vécu des expériences différentes dans leurs activités de littératie familiale, le niveau d'utilisation du français à la maison a été mesuré à l'aide d'un questionnaire comprenant 8 énoncés portant sur la langue parlée à l'enfant par les parents, les frères, les sœurs et la gardienne (jour et soir) ainsi que pendant les activités de lecture et l'écoute de produits médiatiques (émissions de télévision et vidéocassettes). Les parents devaient indiquer la fréquence d'utilisation du français sur une échelle à 5 niveaux (jamais, rarement, parfois, souvent, toujours). Ce questionnaire a été rempli par les parents à la fin de la maternelle et de la $2^{\mathrm{e}}$ année. 
Tableau 1

Caractéristiques et informations sur les participants à l'étude

\begin{tabular}{|c|c|c|c|c|}
\hline & $\begin{array}{l}\text { Programme à } \\
\text { mi-temps } \\
\text { maternelle }\end{array}$ & $\begin{array}{l}\text { Programme à } \\
\text { temps plein } \\
\text { maternelle }\end{array}$ & $\begin{array}{l}\text { Programme à } \\
\text { mi-temps } \\
\text { en } 2^{\mathrm{e}} \text { année }\end{array}$ & $\begin{array}{l}\text { Programme } \\
\text { à temps plein } \\
\text { en } 2^{\mathrm{e}} \text { année }\end{array}$ \\
\hline $\begin{array}{l}\text { Nombre d'élèves } \\
\text { HNUF } \\
\text { FNUF }\end{array}$ & $\begin{array}{l}92 \\
40\end{array}$ & $\begin{array}{c}116 \\
32\end{array}$ & $\begin{array}{l}61 \\
32\end{array}$ & $\begin{array}{l}98 \\
29\end{array}$ \\
\hline $\begin{array}{l}\text { Âge moyen des élèves (en mois) } \\
\text { HNUF } \\
\text { FNUF }\end{array}$ & $\begin{array}{l}59,1 \\
58,9\end{array}$ & $\begin{array}{l}59,4 \\
59,1\end{array}$ & $\begin{array}{l}95,1 \\
94,9\end{array}$ & $\begin{array}{l}95,4 \\
95,1\end{array}$ \\
\hline $\begin{array}{l}\text { Nombre d'élèves dont le français est } \\
\text { la langue maternelle } \\
\text { HNUF } \\
\text { FNUF }\end{array}$ & $\begin{array}{c}75 \\
7\end{array}$ & $\begin{array}{r}94 \\
9\end{array}$ & $\begin{array}{c}48 \\
6\end{array}$ & $\begin{array}{c}78 \\
8\end{array}$ \\
\hline $\begin{array}{l}\text { Sexe } \\
\text { Filles HNUF } \\
\text { Garçons HNUF }\end{array}$ & $\begin{array}{l}47 \\
45\end{array}$ & $\begin{array}{l}64 \\
52\end{array}$ & $\begin{array}{l}31 \\
30\end{array}$ & $\begin{array}{l}54 \\
44\end{array}$ \\
\hline $\begin{array}{l}\text { Filles FNUF } \\
\text { Garçons FNUF }\end{array}$ & $\begin{array}{l}24 \\
16\end{array}$ & $\begin{array}{l}13 \\
19\end{array}$ & $\begin{array}{l}14 \\
18\end{array}$ & $\begin{array}{l}14 \\
15\end{array}$ \\
\hline $\begin{array}{l}\text { Nombre d'élèves membres d'une } \\
\text { minorité raciale } \\
\text { HNUF } \\
\text { FNUF }\end{array}$ & $\begin{array}{c}11 \\
0\end{array}$ & $\begin{array}{l}9 \\
0\end{array}$ & $\begin{array}{c}10 \\
0\end{array}$ & $\begin{array}{l}9 \\
0\end{array}$ \\
\hline $\begin{array}{l}\text { Nombre de parents répondants } \\
\text { HNUF } \\
\text { FNUF }\end{array}$ & $\begin{array}{l}77 \\
31\end{array}$ & $\begin{array}{c}105 \\
26\end{array}$ & $\begin{array}{l}57 \\
21\end{array}$ & $\begin{array}{l}80 \\
26\end{array}$ \\
\hline $\begin{array}{l}\text { Pourcentage de mères ayant un } \\
\text { diplôme universitaire } \\
\text { HNUF } \\
\text { FNUF }\end{array}$ & $\begin{array}{l}50,7 \% \\
16,1 \% \\
\end{array}$ & $\begin{array}{l}50,4 \% \\
15,4 \% \\
\end{array}$ & $\begin{array}{l}54,6 \% \\
27,2 \% \\
\end{array}$ & $\begin{array}{l}51,9 \% \\
10,0 \% \\
\end{array}$ \\
\hline $\begin{array}{l}\text { Pourcentage de pères ayant un } \\
\text { diplôme universitaire } \\
\text { HNUF } \\
\text { FNUF }\end{array}$ & $\begin{array}{l}46,7 \% \\
19,4 \%\end{array}$ & $\begin{array}{c}43,0 \% \\
0,0 \%\end{array}$ & $\begin{array}{l}52,6 \% \\
27,3 \%\end{array}$ & $\begin{array}{c}44,8 \% \\
5,3 \%\end{array}$ \\
\hline
\end{tabular}

Le tableau 2 présente les moyennes obtenues aux échelles d'utilisation du français à la maison pour les élèves à mi-temps et ceux à temps plein, aux deux étapes. Les analyses de variance (ANOVA) confirment qu'à la fin de la maternelle, il n'existe pas de différence significative entre les moyennes des sous-groupes HNUF à mi-temps $(31,7)$ et à temps plein $(30,8)(F[3,135]=0,68 ; n s)$, ni entre les deux sous-groupes FNUF à mi-temps $(21,0)$ et à temps plein $(19,8)(F[3,66]=1,44 ; n s)$. À la fin de la 2e année, il n'existe toujours pas de différence entre les sous-groupes HNUF à mi-temps $(33,0)$ et HNUF à temps plein $(31,4)(F[3,126]=1,4 ; n s)$, ni entre les sous-groupes FNUF qu'il soit à mi-temps $(21,7)$ ou à temps plein $(17,7)(F[1,34]=1,9 ; n s)$. Ainsi, la moyenne d'utilisation du français à la maison pour chacun des deux groupes HNUF et FNUF comparés à eux-mêmes est restée stable de la fin de la maternelle à la fin de la $2^{\mathrm{e}}$ année.

Cependant, lorsque les deux groupes sont comparés entre eux, des différences significatives se révèlent à la fin de la maternelle entre les groupes $\operatorname{HNUF}(31,7)$ et $\operatorname{FNUF}(21,0)$ ayant suivi le programme quatre ans à mi-temps $(F[1,108]=52,34 ; p<, 001)$ de même qu'entre les groupes $\operatorname{HNUF}(30,8)$ et $F N U F(19,8)$ ayant suivi ce programme à temps plein $(F[1,94]=31,53 ; p<, 0001)$. Une différence significative existe aussi à la fin de la $2^{\mathrm{e}}$ année entre les 
groupes $\operatorname{HNUF}(33,0)$ et $F \operatorname{NUF}(21,7)$ qui ont suivi le programme à mi-temps $(F[1,70] 39,54 ; p<, 0001)$ et les groupes HNUF $(31,4)$ et FNUF $(17,7)$ l'ayant suivi à temps plein ( $F[1,90]=42,6 ; p<, 0001)$. Ces analyses confirment donc l'existence de deux groupes distincts au niveau de l'utilisation du français à la maison (HNUF et FNUF). Elles confirment également le maintien du niveau d'utilisation du français entre la maternelle et la $2^{\mathrm{e}}$ année au sein de ces deux groupes.

Tableau 2

\section{Moyennes obtenues à l'échelle d'utilisation du français à la maison par les deux groupes de sujets}

\begin{tabular}{|l|c|c|c|c|}
\hline \multirow{2}{*}{ Groupes } & \multicolumn{2}{|c|}{ HNUF } & \multicolumn{2}{c|}{ FNUF } \\
\cline { 2 - 5 } & Maternelle & $2^{\mathrm{e}}$ année & Maternelle & $2^{\mathrm{e}}$ année \\
\hline Mi-temps & 31,7 & 33,0 & 21,0 & 21,7 \\
\hline Temps plein & 30,8 & 31,4 & 19,8 & 17,7 \\
\hline
\end{tabular}

\section{La procedure et les instruments de collecte de donnée}

La collecte des données a eu lieu pendant les deux dernières semaines de mai de chaque année afin d'assurer un temps de développement identique aux deux groupes de sujets évalués. Quatre étudiantes inscrites à la maîtrise en orthophonie ont procédé à l'évaluation du langage des enfants. Une consultante en orthophonie a offert à celles-ci une formation de deux jours. Cette orthophoniste a également supervisé l'administration des tests et l'interprétation des résultats. Les parents et les enseignantes ont complété leurs questionnaires au cours de la même période.

Les données recueillies portent sur le langage et le rendement en lecture des élèves. Le test utilisé pour l'évaluation du langage à la fin de la maternelle et de la $2^{\mathrm{e}}$ année est l'Échelle de vocabulaire en images Peabody (ÉVIP) (Dunn, Thériault-Whalen et Dunn, 1993). L'ÉVIP évalue l'étendue du vocabulaire réceptif en français. Les enfants doivent montrer du doigt, parmi un choix de quatre images, celle qui illustre un mot prononcé par l'examinateur. Ce test inclut des normes de performance francophones pancanadiennes.

L'évaluation du rendement en lecture porte, à la première étape, sur la conscience de l'écrit mesurée à l'aide des échelles de l'Instrument de mesure du développement de la petite enfance (Centre canadien d'études sur les enfants à risque, 1999) remplies par les enseignantes. Cette évaluation touche les aspects fonctionnels et formels de l'écrit. L'échelle d'évaluation des aspects fonctionnels inclut 6 énoncés qui visent à vérifier si l'enfant comprend l'utilité de la lecture et de l'écriture et s'il différencie les illustrations du texte. Les aspects formels de l'écrit sont évalués par 9 énoncés qui touchent principalement la capacité de lire et d'écrire des lettres, des mots et des phrases.

Au cours de la seconde étape, deux instruments servent à évaluer la lecture : le Test de compréhension de la lecture Le lion (Conseil des écoles catholiques de langue française du Centre-Est de l'Ontario, 2001) et le Test de fluidité de la lecture (Conseil scolaire de district catholique de l'Est de l'Ontario, 2001). Pour le test de compréhension de la lecture, l'élève lit un texte de 400 mots et répond par écrit à dix questions de compréhension. Pour le test de 
fluidité, l'élève lit un texte à haute voix pendant une minute. Les résultats de ce dernier test tiennent compte du nombre de mots lus par minute, de la qualité de la lecture orale et du pourcentage de méprises.

\section{Le plan d'analyse des données}

La présente étude a pour but, rappelons-le, de répondre à deux questions de recherche. La première s'intéresse aux effets d'un programme de maternelle quatre ans à temps plein, comparativement à un programme à mi-temps, sur le développement du langage et de la lecture d'un groupe d'élèves dont le milieu familial démontre un haut niveau d'utilisation du français dans ses activités de littératie (HNUF) et sur celui d'un groupe d'élèves dont le milieu familial utilise peu le français (FNUF). Pour répondre à cette question, nous avons comparé les moyennes obtenues par les élèves des sous-groupes à mi-temps et à temps plein aux tests de langage et de lecture à la fin de la maternelle et de la $2^{\mathrm{e}}$ année, pour chacun des deux groupes FNUF et HNUF. Les moyennes de ces groupes ont été soumises à des analyses de variance ANOVA.

La deuxième question vise à savoir si le programme de maternelle quatre ans à temps plein permet aux élèves du groupe FNUF de rejoindre la performance en langage et en lecture des élèves du groupe HNUF. Pour y répondre, nous avons comparé les moyennes obtenues aux tests de langage et de lecture, à la fin de la maternelle et de la $2^{\mathrm{e}}$ année, par les élèves des sous-groupes cibles FNUF et HNUF qui ont fréquenté le programme de maternelle à temps plein. Les moyennes de ces groupes ont été soumises aux mêmes analyses statistiques que celles utilisées pour répondre à la première question.

\section{Les résultats}

Le tableau 3 présente les moyennes obtenues par les différents groupes d'élèves en langage réceptif et en lecture. Pour la première question portant sur les effets du programme, les sous-groupes à mi-temps et à temps plein dont le milieu familial démontre un haut niveau d'utilisation du français dans ses activités de littératie (HNUF) ont obtenu respectivement, en langage, à la fin de la maternelle, un rang centile de 45,6 et de 42,3 ( $F[3,139]=2,24$; ns). À la fin de la 2e année, le sous-groupe à mi-temps a obtenu un rang centile de 74,7 et celui à temps plein, de 71,7 (F $[3,126]=0,48 ; n s)$. Ces résultats indiquent que le programme à temps plein, comparativement au programme à mitemps, n'a pas produit d'effet important en langage réceptif pour le groupe HNUF, tant à la fin de la maternelle qu'à la fin de la $2^{e}$ année. En effet, en trois ans, l'écart des rangs centiles entre les élèves des sous-groupes HNUF ayant suivi le programme à mi-temps et à temps plein est sensiblement le même, aux environs de 29 rangs centiles.

Pour leur part, à la fin de la maternelle, les sous-groupes d'élèves fréquentant les programmes à mi-temps et à temps plein, et dont le milieu familial utilise peu le français dans ses activités de littératie (FNUF), ont obtenu respectivement, en langage réceptif, un rang centile de 9,3 et de $17,3(F[3,59]=3,2 ; p<, 01)$. À la fin de la $2^{e}$ année, le sous-groupe à mi-temps a obtenu un rang centile de 41,7 et celui à temps plein, de 63,8 (F [1,34] = 4,16; $p<, 05)$. Ainsi, pour le groupe FNUF, les résultats indiquent que le programme à temps plein, comparativement au programme à mi-temps, a produit un effet important en langage réceptif tant à la fin de la maternelle qu'à la fin de la $2^{\mathrm{e}}$ année. L'écart en rangs centiles est plus grand pour les élèves ayant suivi le programme à temps plein $(46,4)$ que pour ceux l'ayant suivi à mi-temps $(32,4)$. 
En lecture, à la fin de la maternelle, les résultats révèlent un effet positif pour les deux groupes cibles en conscience de l'écrit, tant pour les aspects fonctionnels que formels. Pour les aspects fonctionnels, les élèves du sousgroupe HNUF à mi-temps ont obtenu une moyenne de 13,0 et ceux à temps plein, une moyenne de 15,8 (F[3,139] = 16,95; $p<$,0001). Pour le groupe FNUF, les élèves à mi-temps ont obtenu une moyenne de 12,3 et ceux à temps plein, une moyenne de 15,8 (F [3,59] = 13,99; $p<$,0001). Pour les aspects formels, le sous-groupe HNUF à mi-temps a obtenu une moyenne de 6,7 et celui à temps plein, une moyenne de 10,9 (F [3,139] = 27,19; $p<, 0001)$, alors que le sous-groupe FNUF à mi-temps a obtenu une moyenne de 7,0 et celui à temps plein, une moyenne de 11,0 (F [3,59] = $13,30 ; p<, 0001)$. À la fin de la $2^{\mathrm{e}}$ année, pour le groupe d'élèves HNUF, le programme à temps plein a eu un effet positif en compréhension de la lecture, les moyennes se situant à 24,2 pour le sous-groupe à mi-temps et à 28,3 pour celui à temps plein $(F[3,126]=3,55 ; p<, 05)$; par contre, ce programme a eu peu d'effets sur la qualité de la lecture, le sous-groupe à mi-temps obtenant une moyenne de 6,8 et celui à temps plein, une moyenne de $6,4(F[3,126]=2,06$; ns). Pour le groupe d'élèves FNUF, l'inverse se produit. Le programme à temps plein a eu un effet positif sur la qualité de la lecture : moyenne de 5,2 pour le sous-groupe à mi-temps et de 7,2 pour celui à temps plein $(F[1,34]=14,1 ; p<$ 01); mais l'effet n'est pas significatif sur la compréhension de la lecture : moyenne de18,8 pour le sous-groupe à mitemps et de 23,5 pour celui à temps plein $(F[1,34]=0,21 ; n s)$.

En somme, en réponse à la première question, les résultats indiquent que le programme de maternelle à temps plein a des effets positifs différentiels pour les deux groupes HNUF et FNUF. Bien qu'elle ne produit pas d'effet particulier sur le développement du langage réceptif, l'augmentation du temps d'exposition aux activités de littératie scolaire semble liée à une conscience de l'écrit et une compréhension en lecture accrues chez les élèves du groupe HNUF. Pour les élèves du groupe FNUF, des retombées positives se révèlent tant au niveau du langage réceptif, qu'à celui du langage écrit, notamment en ce qui a trait à la conscience de l'écrit et à la qualité de la lecture.

Tableau 3

\section{Moyennes obtenues en langage et en lecture par les deux groupes de sujets}

\begin{tabular}{|c|c|c|c|c|c|}
\hline & & \multicolumn{2}{|c|}{$\begin{array}{c}\text { Haut niveau d'utilisation } \\
\text { du français }\end{array}$} & \multicolumn{2}{|c|}{$\begin{array}{c}\text { Faible niveau d'utilisation } \\
\text { du français }\end{array}$} \\
\hline & & Fin maternelle & Fin 2e année & Fin maternelle & Fin 2e année \\
\hline \multicolumn{6}{|l|}{ Langage } \\
\hline \multirow{2}{*}{ ÉVIP (rangs centiles) } & Mi-temps & 45,6 & 74,7 & 9,3 & 41,7 \\
\hline & Temps plein & 42,3 & 71,7 & 17,4 & 63,8 \\
\hline \multicolumn{6}{|l|}{ Lecture } \\
\hline \multirow{2}{*}{ Qualité de la lecture } & Mi-temps & NA & 6,8 & NA & 5,2 \\
\hline & Temps plein & NA & 6,4 & NA & 7,2 \\
\hline \multirow{2}{*}{$\begin{array}{l}\text { Compréhension de la lecture } \\
\text { (max. 40) }\end{array}$} & Mi-temps & NA & 24,2 & NA & 18,8 \\
\hline & Temps plein & NA & 28,3 & NA & 23,5 \\
\hline \multirow{2}{*}{ Aspects formels } & Mi-temps & 6,7 & NA & 7,0 & $\mathrm{NA}$ \\
\hline & Temps plein & 10,9 & NA & 11,0 & NA \\
\hline \multirow{2}{*}{ Aspects fonctionnels } & Mi-temps & 13,0 & NA & 12,3 & NA \\
\hline & Temps plein & 15,8 & NA & 15,8 & NA \\
\hline
\end{tabular}

NA : ne s'applique pas à cause de l'âge des enfants 
L'analyse des résultats en réponse à la deuxième question qui porte sur l'effet compensateur du programme, indique que même si le programme de maternelle quatre ans à temps plein a eu un effet positif en langage réceptif pour les élèves dont le milieu familial utilisait peu le français (FNUF), aux deux étapes de l'évaluation, le programme ne leur permet pas d'atteindre le niveau de performance des élèves HNUF. En effet, les élèves FNUF qui ont fréquenté le programme à temps plein ont obtenu un rang centile de 63,8 à la fin de la 2e année alors que celui obtenu par le groupe HNUF est de 71,7 (F $[1,89]=4,17 ; p<, 05)$.

En lecture, à la fin de la maternelle, le programme à temps plein a permis au groupe d'élèves FNUF de rejoindre la performance en conscience de l'écrit du groupe d'élèves HNUF. En effet, les moyennes pour les aspects fonctionnels sont identiques pour ces deux groupes, se situant à 15,8 ( $F[3,10]=0,14$; ns). Pour les aspects formels, les moyennes sont de 11,0 et de 10,9 respectivement $(F[3,101]=0,27 ; n s)$. À la fin de la 2e année, le programme a compensé pour la qualité de la lecture, les élèves du groupe FNUF obtenant une moyenne de 7,2 et ceux du groupe HNUF, une moyenne de 6,4 ( $F[1,89]=2,17$; ns). Cependant, le programme ne semble pas compenser pour la compréhension en lecture, les moyennes étant, pour le groupe FNUF, de 23,5 et pour le groupe HNUF, de 28,3 (F $[1,89]=4,04 ; p<, 05)$.

\section{Discussion des résultats}

Les résultats indiquent que le groupe d'élèves dont le milieu familial utilise peu le français dans ses activités de littératie (FNUF) a bénéficié du programme de maternelle quatre ans à temps plein tant sur le plan du langage réceptif que sur celui de la lecture et ce, autant à la fin de la maternelle qu'à la fin de la $2^{\mathrm{e}}$ année. En langage, le progrès réalisé par ce groupe permet de supposer que le programme à temps plein a compensé, en partie, une littératie familiale qui utilise peu le français. La participation au programme ne permet cependant pas à ces élèves de rejoindre ceux dont le milieu familial démontre un haut niveau d'utilisation du français dans ses activités de littératie (HNUF). Chez ces derniers, les progrès en langage réceptif apparaissent moins importants. En effet, le programme a eu peu d'effet différentiel sur le langage de ceux qui ont fréquenté le programme à mi-temps et ceux qui l'ont fréquenté à plein temps. De plus, le progrès réalisé entre la fin de la maternelle et la fin de la $2^{\mathrm{e}}$ année par les élèves qui ont fréquenté le programme à temps plein est beaucoup moins important que celui des élèves dont le milieu familial utilise peu le français dans ses activités de littératie (FNUF). En lecture, le programme semble avoir compensé pour les élèves FNUF à la fin de la maternelle, leur permettant ainsi de rejoindre le groupe HNUF pour les aspects fonctionnels et formels de la conscience de l'écrit. Cependant, à la fin de la $2^{e}$ année, malgré des progrès en qualité et en compréhension de la lecture chez les élèves du groupe FNUF, le programme a compensé pour la qualité de la lecture mais non pour la compréhension en lecture.

Ces résultats semblent concorder avec ceux rapportés antérieurement dans la recension des écrits selon lesquels les programmes préscolaires de qualité ont des effets positifs sur le langage à court terme ou ultérieurement, et les élèves des milieux défavorisés et ceux appartenant à une minorité visible ou raciale en bénéficient davantage que les élèves provenant de milieux favorisés. Les résultats de la présente recherche en arrivent sensiblement aux mêmes conclusions, mais pour les élèves qui vivent en contexte linguistique minoritaire. En langage et en lecture, le programme de maternelle quatre ans à temps plein a produit des effets positifs pour les deux groupes HNUF et FNUF, 
mais les effets sont plus importants pour les élèves dont le milieu familial utilise peu le français dans ses activités de littératie (FNUF).

Ces résultats nous amènent à poser quelques questions : comment se fait-il qu'un programme quatre ans à temps plein produise un effet moins important sur le langage des élèves dont le milieu familial démontre un haut niveau d'utilisation du français dans ses activités de littératie (HNUF)? Plusieurs hypothèses peuvent être mises de l'avant. À la lumière des résultats, l'utilisation du français à la maison est restée stable tant chez les élèves qui ont fréquenté le programme à mi-temps que ceux qui l'ont fréquenté à temps plein. La famille a donc maintenu le même niveau d'utilisation du français dans ses activités de littératie. Du côté scolaire, selon l'orthophoniste qui a supervisé le projet et qui a collaboré au programme de dépistage précoce du conseil scolaire pendant de nombreuses années, pour produire des effets plus importants chez les élèves HNUF, il aurait fallu réellement complexifier le programme au plan langagier (vocabulaire, structure de phrase, structure des types de situation langagière). Étant donné qu'ils parlent français en arrivant à l'école, le programme prescrit par le ministère de l'Éducation de l'Ontario devrait probablement être enrichi. Une autre hypothèse a trait à la composition des classes du préscolaire qui sont composées d'élèves qui parlent français et d'autres qui ne sont pas nécessairement à l'aise dans cette langue. Le personnel enseignant utilise des stratégies particulières pour franciser les élèves qui ne parlent pas beaucoup le français qui ne s'appliquent pas nécessairement aux élèves dont le milieu démontre un haut niveau d'utilisation du français dans ses activités de littératie familiale (HNUF). Loin de prôner la formation de classes homogènes, il serait souhaitable de réexaminer les stratégies de francisation et de voir comment elles pourraient s'inscrire davantage en complémentarité avec les stratégies d'enrichissement du langage des élèves du groupe HNUF, afin que les programmes de maternelle quatre ans temps plein répondent aux besoins de tous les élèves.

Une autre question à se poser : quelles sont les stratégies à mettre en place pour permettre aux élèves dont le milieu familial utilise peu le français dans ses activités de littératie (FNUF) de rattraper ceux dont le milieu familial démontre un haut niveau d'utilisation du français dans ses activités de littératie (HNUF)? Bien que le programme quatre ans à temps plein ait permis des gains significatifs en langage et en lecture pour les élèves FNUF, I'utilisation du français à la maison s'est maintenue pour les élèves qui l'avaient fréquenté à temps plein. Tout comme le mentionnent Burgess, Hecht et Lonigan (2002), les caractéristiques démographiques et sociales de l'environnement influencent le développement de la littératie de l'enfant. En contexte minoritaire, ces caractéristiques pèsent lourd sur la réussite des élèves. Le milieu scolaire doit donc mettre en place certaines pratiques de littératies multiples afin de répondre aux besoins de ses élèves. II doit exercer un leadership en travaillant en partenariat avec le milieu familial et la communauté pour encourager l'utilisation du français dans les activités de la vie quotidienne des élèves. II doit s'engager avec les organismes communautaires francophones à fournir des activités variées pour les parents et les enfants avant même que ces derniers ne fréquentent le milieu scolaire. À titre d'exemple, les centres d'alphabétisation de plusieurs régions de l'Ontario offrent des programmes d'activités qui favorisent le développement langagier et l'éveil à l'écrit chez les enfants (Letouzé, 2006).

Il est surprenant de constater que même si le niveau d'utilisation du français à la maison s'est maintenue à la fin de la $2^{\mathrm{e}}$ année, les élèves FNUF ayant fréquenté le programme à temps plein ont réalisé des progrès importants en 
langage et en lecture. Un programme quatre ans à temps plein de qualité contribue donc à combler, en partie, le faible niveau d'utilisation du français du milieu familial dans ses activités de littératie. Cependant, il confirme aussi que les activités de littératie familiale, telles que la lecture fréquente de livres faite aux enfants, les discussions langagières parent-enfant au cours de ces lectures, l'écoute d'émissions de télévision ou de vidéocassettes, contribuent au succès scolaire des enfants (Burgess, Hecht et Lonigan, 2002; Bus, Van ljzendoorn et Pellegrini, 1995; Giasson, 2003). Des recherches ultérieures pourraient être menées afin de vérifier l'incidence des activités familiales en littératie lorsqu'elles sont vécues dans une langue autre que celle de l'enseignement. Est-ce que les apprentissages en littératie réalisés dans une autre langue par les enfants se transfèrent dans la langue d'enseignement?

En conclusion, pour la minorité de langue française de l'Ontario, la participation à un programme de maternelle quatre ans à temps plein produit des effets positifs chez les deux groupes d'élèves, indépendamment du fait qu'ils proviennent d'un milieu familial démontrant un haut niveau d'utilisation du français dans ses activités de littératie ou d'un milieu familial qui utilise peu le français dans ses activités. Les effets sont cependant davantage importants pour ces derniers. Les conseils scolaires de langue française doivent donc se pencher sur les moyens à prendre afin que le programme quatre ans à temps plein réponde aux besoins particuliers de tous les élèves tant pour ceux dont le milieu démontre un haut niveau d'utilisation du français dans ses activités de littératie familiale (HNUF) que pour ceux dont le niveau d'utilisation du français est faible (FNUF). Pour y arriver, des recherches ultérieures devraient être menées afin de trouver des stratégies particulières à développer en milieu scolaire, familial et communautaire pour ces deux groupes d'élèves.

\section{Références}

Adams, M. J. (1990). Beginning to read : Thinking and learning about print. Cambridge, MA : MIT Press.

Burgess, S. R., Hecht, S. A. et Lonigan, C. J. (2002). Relations of the home literacy environment (HLE) to the developmental reading-related abilities : A one-year longitudinal study. Reading Research Quarterly, 37(4), 408-426.

Burns, S., Espinosa, L. et Snow, C. E. (2003). Débuts de la littératie, langue et culture : perspective socioculturelle. Revue des Sciences de l'Éducation, 29(1), 75-100.

Bus, A. G., Van ljzendoorn, M. H. et Pellegrini, A. D. (1995). Joint book reading makes for success in learning to read : A meta analysis on intergenerational transmission of literacy. Review of Educational Research, 65(1), 1-21.

Centre canadien d'études sur les enfants à risque (1999). Instruments de mesure du développement de la petite enfance (IMDPE). Hamilton, ON : Université McMaster, Hamilton Sciences Corporation.

Comber, B. et Cormack, P. (1997). Looking beyond skills and processes : Literacy as social and cultural practices in classrooms. Reading, 31(3), 22-29.

Conseil des écoles catholiques du Centre-Est de l'Ontario (2001). Test de compréhension de la lecture : $2^{e}$ année. Ottawa, ON : Conseil des écoles catholiques du Centre-Est de l'Ontario.

Conseil des écoles catholiques de l'Est de l'Ontario (2001). Test de fluidité de la lecture. Ottawa, ON : Conseil des écoles catholiques de l'Est de l'Ontario.

Dunn, L. M., Thériault-Whalen, C. M. et Dunn, L. M. (1993). Échelle de vocabulaire en images Peabody (ÉVIP). Adaptation française du Peabody Picture Vocabulary Test - Revised. Toronto, ON : Psycan.

Enz, B. J. (2003). The ABCs of family literacy. In A. DeBruin-Parecki et B. Krol-Sinclair (Eds.), Family literacy : From theory to practice (p. 50-67). Newark, DL : International Reading Association.

Giasson, J. (2003). La lecture : de la théorie à la pratique. Montréal, QC : Gaëtan Morin.

Jarousse, J.-P., Mingat, A. et Richard, M. (1992). La scolarisation à 2 ans : effets pédagogiques et sociaux. Éducation et formations, 31, 3-9.

Jeantheau, J.-P. et Murat, F. (1998). Observation à l'entrée au CP des élèves du « panel 1997 ». Note d'information du ministère de l'Éducation nationale, de la Recherche et de la Technologie, 98.40 (décembre). 
Letouzé, S. (2006). Pour mon enfant d'abord : étude de l'impact de l'alphabétisation familiale sur les familles vivant en milieu minoritaire : Étape 2 : Cohortes 2 et 3. Ottawa, ON : Coalition francophone pour l'alphabétisation et la formation de base en Ontario.

Maltais, C. (2005). Relation entre les types de services de garde et le développement du langage chez les enfants du préscolaire. Éducation et francophonie, 33(2), 207-223.

Maltais, C. et Herry, Y. (2005). Un programme 4 ans à temps plein : ça compte encore! Évaluation du programme à temps plein destiné aux enfants de 4 ans. Ottawa, ON : Conseil des écoles catholiques de langue française du Centre-Est.

Maltais, C., Herry, Y. et Levesque, D. (2001). Un programme 4 ans à temps plein : ça compte! Ottawa, ON : Conseil des écoles catholiques de langue française du Centre-Est.

Masny, D. (2001). La culture de l'écrit : les défis à l'école et au foyer. Montréal, QC : Les Éditions Logiques.

Meyer, L. A., Wardrop, J. L., Stahl, S. A. et Linn, R. L. (1994). Effects of reading storybooks aloud to children. Journal of Educational Research, 88(2), 69-85.

O'Brien Caughy, M., Dipietro, J. A. et Scrobino, M. (1994). Day-care participation as a projective factor in the cognitive development of low-income children. Child Development, 65(2), 457-471.

Palacio-Quintin, E. et Coderre, R. (1999). Les services de garde à l'enfance : influence des différents types de garde sur le développement de l'enfant. Trois-Rivières, QC : Université du Québec à Trois-Rivières, Rapport présenté au Conseil Québécois de la Recherche Sociale.

Scarborough, H. S. et Dobrich, W. (1994). On the efficacy of reading preschoolers. Developmental Review, 14(3), 245-302.

Sénéchal, M., Lefevre, J. A., Thomas, E. M., et Daley, K. E. (1998). Differential effects of home literacy experiences on the development of oral and written language. Reading Research Quaterly, 33(1), 96-116

Sénéchal, M., Thomas, E. et Monker, J.-A. (1995). Individual differences in vocabulary acquisition. Journal of Educational Psychology, 87(2), 218-229.

Thériault, J. (1995). J'apprends à lire... Aidez-moi! Montréal, QC : Les Éditions Logiques.

Tremblay, R. E. (2003). Grandir en qualité : recension des écrits sur la qualité des services de garde. Québec, QC : Ministère de la Famille et de l'Enfance, Direction de la recherche, de l'évaluation et de la statistique.

Walker, D., Greenwood, C., Hart, B. et Carta, J. (1994). Prediction of school outcomes based on early language production and socioeconomic factors. Child Development, 65(2), 606-621.

Weigel, D. J., Martin, S. S. et Bennett, K. K. (2005). Ecological influences of the home and the child-care center on preschoolage children's literacy development. Reading Research Quarterly, 40(2), 204-233.

Wessels, H., Lamb, M. E. et Hwang, C.-P. (1996). Cause and causality in daycare research: An investigation of group differences in Swedish child care. European Journal of Education, 11(2), 231-245.

Whitehurst, G. J., Epstein, J. N., Angell, A. L., Payne, A. C., Crone, D. A. et Fishel, J. E. (1994). Outcomes of an emergent literacy intervention in Head Start. Journal of Educational Psychology, 86(4), 542-555. 